\title{
O reconhecimento de expressões faciais e prosódia emocional: Investigação preliminar em uma amostra brasileira jovem.
}

\author{
Hélida Arrais Costa-Vieira \\ Wânia Cristina de Souza \\ Universidade de Brasília
}

\begin{abstract}
Resumo
Sinais não verbais de comunicação emocional são frequentes em diferentes culturas. Esta pesquisa buscou traduzir e adaptar uma bateria (Florida Affect Battery) de avaliação de expressões faciais e prosódia emocional, bem como avaliar o desempenho de uma amostra populacional brasileira em seus subtestes, incluindo uma comparação entre participantes do sexo feminino e masculino e por escolaridade. Os resultados da amostra brasileira foram avaliados e, posteriormente, comparados aos da amostra estadunidense, fornecidos no manual da bateria. Não foram encontradas diferenças estatisticamente significantes entre o desempenho da amostra brasileira e da amostra estadunidense, bem como entre homens e mulheres, com altas taxas de acerto para todos os grupos avaliados, evidenciando um bom reconhecimento de expressões faciais e prosódia emocional.
\end{abstract}

Palavras-chave: expressões faciais; percepção da face; percepção da fala; disprosodia emocional; Florida affect battery.

\begin{abstract}
Recognition of emotional facial expressions and of emotional prosody: A preliminary investigation in a young brazilian sample. Nonverbal signs of emotional communication are common in different cultures. This study aimed to translate and adapt a battery (Florida Affect Battery) of evaluation of facial expressions and emotional prosody, as well as evaluate the performance of a Brazilian sample in their subtests, including a comparison of men and women and of education level. The results of the Brazilian sample were compared to the results of United States sample provided in battery's manual. There were no significant differences between the performance of Brazilian sample and the United States sample as well as between men and women, with high success rates for all groups evaluated.
\end{abstract}

Keywords: facial expressions; face perception; speech perception, emotional disprosody; Florida affect battery.

\section{Resumen}

El reconocimiento de las expresiones faciales y la prosodia emocional: Investigación preliminar en una muestra brasileña joven. Señales no verbales de comunicación emocional son comunes em diferentes culturas. Este estudio tuvo como objetivo traducir y adaptar una batería (Florida Affect Battery) de evaluación de expresiones faciales y prosodia emocional, así como evaluar el desempeño de una muestra de la población brasileña en sus subpruebas, incluyendo uma comparación entre participantes hombres y mujeres e entre los diferentes níveles de educación. Los resultados de la muestra brasileña fueron evaluados, y posteriormente, se compararon con los resultados de la muestra estadounidense, proporcionados en el manual de la batería. No hubo diferencias significativas entre el desempeño de la muestra brasileña y la muestra estadunidense, así como entre hombres y mujeres, con altas tasas de acierto para todos los grupos evaluados, evidenciando un buen reconocimiento de expresiones faciales y prosodia emocional.

Palabras clave: expresiones faciales; percepción facial; percepción del habla; disprosodia emocional, Florida affect battery.

$\mathrm{E}$ moções básicas são respostas afetivas pré-programadas evolutivamente, expressas e reconhecidas de modo semelhante por toda uma espécie, constituídas de respostas fisiológicas distintas para situações de sobrevivência e fundamentais para a vida (Tracy \& Randles, 2011). Estas respostas são universalmente compartilhadas e algumas podem ser encontradas em primatas e outros mamíferos (Ekman, 1992; Ekman \& Cordaro, 2011; Izard, 
2010). Darwin (1998) foi um dos primeiros pesquisadores em emoções básicas a alertar para a pluralidade de formas de expressão emocional e também a investigar estes fenômenos em diferentes culturas. Ekman e Friesen (1969) prosseguiram estes estudos transculturais, investigando diferentes sinais nãoverbais de comunicação (expressões faciais, gestos e postura corporal) em busca de informações acerca de significados, funções, origens, categorizações, atribuindo às expressões faciais a função de transmitir emoções de forma universal.

Ao longo dos anos, expressões faciais e prosódia emocional foram mais enfatizadas pelas pesquisas científicas (Campanella \& Bellin, 2007; Paulman, Titone, \& Pell, 2012; Rigoulout \& Pell, 2012), em detrimento de outros sinais não-verbais de expressão da emoção, como gestos, posturas corporais, movimentos.

É sabido que dentre os diferentes sinais não-verbais de expressão da emoção, as expressões faciais são aquelas com maior destaque, mediando boa parte das interações sociais e da comunicação não-verbal. Através da observação da expressão alheia, pode-se inferir informações sobre o estado emocional de uma pessoa, suas intenções e, inclusive, suas reações aos eventos apresentados em nosso ambiente (Whalen et al., 2013). São consideradas expressões faciais emocionais universais as seguintes: alegria, tristeza, raiva, medo, surpresa e nojo (Ekman, 1999).

Caracterizada como a entonação ou a modulação da voz, a prosódia possui caráter essencial para comunicação humana, acrescentando informações além do contexto semântico transmitido. A voz não carrega apenas a informação semântica, mas também pode comunicar informações através de aspectos para-verbais como os parâmetros acústicos (altura, intensidade, tom) e a prosódia (Regenbogen et al., 2012). A prosódia pode ser não-emocional quando se destina a aspectos formais da linguagem, estabelecidos por convenções, como a entonação característica de uma pergunta ou afirmação, mas também pode incorporar aspectos emocionais, como transmitimos pesar ou tristeza ao passar uma informação com significação triste (Testa, Beatty, Gleason, Orbelo, \& Ross, 2001).

Apesar de também poderem ser classificadas pelas categorias de emoções básicas, ainda não existem estudos conclusivos que apontem indícios fidedignos de universalidade na expressão e no reconhecimento de posturas corporais, de gestual (emblemas) e de prosódia emocional, sendo considerados, geralmente, como construídos culturalmente, tendo seu reconhecimento, significado e interpretação vinculados ao contexto social (Scherer, Banse, \& Wallbott, 2001). No entanto, um reconhecimento bidirecional entre duas culturas (tribo himba - Namíbia e ingleses), dentro das categorias das emoções básicas, foi identificado para vocalizações emocionais como gritos, gargalhadas, murmuros, choros, entre outros (Sauter, Eisner, Ekman, \& Scott, 2010).

Compreender a emoção do outro está associado a questões de relevância e competência social, como interação e empatia, e sofre influência de diferentes fatores, como regras culturais (Ekman, 2003), intimidade com o outro (Ma-Kellams \& Blascovitch, 2012), contexto ambiental e emocional (Marian \& Shimamura, 2012) e até posturas corporais (Dael, Mortillaro, \& Scherer, 2012). Déficits no processamento emocional são identificados como um mecanismo central para dificuldades comportamentais e de interação social, já que os indivíduos acometidos de tais déficits tendem a interpretar equivocadamente as "deixas emocionais" que normalmente ajudam a guiar seus comportamentos, incluindo dificuldades no reconhecimento de expressões faciais e prosódia (Bucks \& Radford, 2004)

Dentre os diferentes instrumentos para avaliação de expressões faciais e prosódia, a Florida Affect Battery (FAB) (Bowers, Blonder, \& Heilman, 1999) se destaca por englobar simultaneamente os dois. Ela foi desenvolvida baseada num modelo cognitivo de "léxico afetivo não-verbal", no qual estruturas cerebrais específicas, primordialmente do hemisfério direito estariam envolvidas em atividades de reconhecimento não-verbal de emoções, compondo um "vocabulário" ou banco de dados, exclusivamente voltados para essa função (Bowers, Bauer, \& Heilman, 1993).

É composta por 11 subtestes, que buscam avaliar questões como reconhecimento de identidade facial, reconhecimento de expressões faciais, reconhecimento de prosódia não-emocional, reconhecimento de prosódia emocional e a interação entre expressões faciais e prosódia emocional. Todos os subtestes foram desenvolvidos para auxiliar no diagnóstico de dificuldades relacionadas ao reconhecimento questões afetivas. A inclusão de aspectos de identidade e da variedade de tarefas para o reconhecimento emocional é uma tentativa de identificar patologias que possam interferir indiretamente e individualmente no reconhecimento de emoções, como a prosopagnosia, a anomia e a aprosodia, de modo a diferenciar dificuldades puramente perceptuais de dificuldades genuinamente afetivas.

Diante da inexistência de um instrumento em língua portuguesa brasileira que avaliasse simultaneamente, de modo sistematizado e organizado, expressões faciais e prosódia, a tradução e adaptação da FAB foi realizada. Um estudo exploratório foi realizado para a verificação da adequação do instrumento, assim como uma avaliação do desempenho da amostra brasileira selecionada no reconhecimento e julgamento de expressões faciais e da prosódia. Também foi realizada uma comparação entre a média de acertos da população brasileira e da população estadunidense, conforme as normas fornecidas no manual da FAB (Bowers et al., 1999). Esta pesquisa foi submetida e aprovada pelo CONEP.

\section{Método}

\section{Participantes}

Participaram desta fase da pesquisa 69 indivíduos de ambos os sexos, sendo 40 mulheres e 29 homens, com idades compreendidas entre 21 e 33 anos, com média de idade de 25,3 anos $(D P=3,34)$ e com escolaridade mínima superior 
incompleto, sendo 46 participantes com superior incompleto, 14 com superior completo e nove com pós-graduação. Como critérios de inclusão foram utilizados a faixa etária, compreendia entre 18 e 35 anos, e a escolaridade mínima de ensino médio completo. Para evitar interferência nas tarefas de reconhecimento emocional, considerou-se como critérios de exclusão: presença de transtornos psiquiátricos, com ênfase nos transtornos afetivos, transtornos neurológicos e dificuldades auditivas ou visuais, exceto quando devidamente corrigidas. Os participantes foram recrutados entre os estudantes de graduação e pós-graduação de faculdades e universidades da cidade de Fortaleza ou por indicação de outros participantes. Não houve nenhum tipo de remuneração ou compensação financeira.

\section{Instrumentos}

As informações referentes a identidade, contato e critérios de inclusão e exclusão foram obtidas através de uma entrevista clínica, realizada com o auxílio de um roteiro estruturado.

A Florida Affect Battery, originalmente em língua inglesa, é composta de 11 subtestes, envolvendo o reconhecimento de expressões faciais (cinco tarefas), prosódia emocional (quatro tarefas) e ambos os estímulos (duas tarefas). Os estímulos das tarefas visuais são realizados por quatro mulheres, cada uma produzindo cinco expressões faciais (alegria, tristeza, raiva, medo e neutro), totalizando 20 expressões diferentes. As tarefas com prosódia são narradas sempre pela mesma pessoa, um vocal feminino, utilizando-se das mesmas cinco emoções. Com exceção do subteste 6 , com 16 itens, e do subteste 8B, com 36 itens, 20 tentativas são apresentadas em cada subteste.

Os 11 subtestes da FAB podem ser sumariamente descritos da seguinte forma:

Subteste 1 - Discriminar identidade facial: nesta tarefa, busca-se estabelecer um controle perceptual para as tarefas afetivas. Duas fotografias, com expressões neutras, são apresentadas por vez e o participante deve dizer se retratam a mesma pessoa ou pessoas diferentes.

Subteste 2 - Diferenciação de afeto facial: duas expressões faciais são apresentadas simultaneamente e os participantes devem determinar se constituem a mesma emoção ou não, buscando estabelecer um controle na diferenciação entre emoções.

Subteste 3 - Nomeação de faces afetivas: expressões faciais são apresentadas senquencial e individualmente e os participantes devem classificá-las, de acordo com as opções: alegria, tristeza, raiva, medo e neutro.

Subteste 4 - Seleção de expressão facial: são apresentadas simultaneamente cinco expressões faciais diferentes para os participantes, por tentativa, e ele deve escolher, dentre as opções, a face adequada de acordo com o comando do examinador (ex. "Aponte a face com raiva").

Subteste 5 - Combinação de expressões faciais: uma face -alvo, com uma expressão emocional específica (ex. alegria), é apresentada como estímulo e o participante deve identificar entre cinco opções de expressões faciais diferentes uma semelhante a face-alvo.

As tarefas envolvendo prosódia foram elaboradas para complementar as tarefas de percepção facial e envolvem entonações emocionais e não-emocionais, bem como conflito entre contexto semântico e entonação de voz adequada.

Subteste 6 - Discriminação de prosódia não emocional: este subteste serve como controle para as tarefas que envolvem entonações afetivas e o participante deve diferenciar, entre os pares de sentenças não-emocionais apresentados, se ambas eram afirmativas, interrogativas ou diferentes.

Subteste 7 - Discriminação de prosódia emocional: duas sentenças são apresentadas e os participantes devem identificar se os pares de sentenças apresentados, com conteúdo verbal-emocional neutro (ex. a garota foi ao mercado), retratam a mesma entonação emotiva ou não (ex. ambos apresentam entonação alegre).

Subteste 8A - Nomeação de prosódia emocional: esta tarefa acessa a habilidade de classificar verbalmente prosódias afetivas. O participante deve nomear a prosódia emocional de cada sentença apresentada, de acordo com as seguintes opções: alegria, tristeza, raiva, medo, neutro.

Subteste 8B - Situações conflitantes: uma frase com mensagem semântica emocional condizente com a entonação esperada ou com a entonação inadequada (ex. todos os cachorrinhos estão mortos em uma entonação alegre) é apresentada e o participante deve nomear a prosódia emocional relacionada, focando-se apenas neste aspecto, abstendo-se da eventual incongruência entre a prosódia e o conteúdo semântico da frase.

Por último, nas tarefas cross-modal (face-prosódia), os participantes devem combinar expressões emocionais faciais com prosódias afetivas e vice-versa.

Subteste 9 - Combinar prosódia emocional com uma expressão facial: apresenta-se uma frase com determinada entonação emocional e o participante deve apontar, dentre três diferentes expressões faciais, aquela correspondente à entonação emocional apresentada pelo locutor.

Subeteste 10 - Combinar uma expressão facial com prosódia emocional: apresenta-se uma face com determinada expressão emocional ao participante e, posteriormente, três diferentes entonações emocionais. O participante deve escolher a sentença com a entonação emocional que melhor combine com a expressão facial anteriormente apresentada.

\section{Procedimento}

Inicialmente, foram realizadas a tradução e adaptação das informações, visto que originalmente o instrumento apresentava-se em língua inglesa, além de verificada a viabilidade de uso dos testes de expressões faciais. As expressões faciais foram avaliadas, seguindo o preceito da universalidade das emoções, bem como qualidade da imagem para visualização. A tradução foi realizada com auxílio e revisão de profissionais bilíngues, observando a adaptação cultural adequada, enfatizando os devidos cuidados nos termos, contextos e prosódia 
emocional associada, sem promover uma desvirtuação do sentido proposto no instrumento original. Para tanto, foram utilizados os procedimentos de tradução direta e tradução reversa, por dois profissionais bilíngües, tendo o inglês como língua-mãe e buscando não somente a equivalência semântica, mas também as equivalências culturais e conceituais (Beaton, Bombardier, Guillemin, \& Ferraz, 2000; International Test Comission, 2010; Organização Mundial de Saúde, 2010). A narração do instrumento traduzido foi conduzida em um estúdio de gravação profissional e realizada por uma atriz de teatro contratada. O material final obtido (estímulos visuais e auditivos) foi avaliado por uma equipe de profissionais, incluindo um fonoaudiólogo, dois psicólogos, dois profissionais do teatro e dois licenciados em Letras Estrangeiras - português/ inglês. Tais procedimentos foram priorizados para se buscar evidências de que a validade de conteúdo havia sido mantida (Hambleton, Merenda, \& Spielperger, 2005).

A aplicação do instrumento traduzido foi conduzido em uma única sessão, de aproximadamente 1 hora de duração, na qual a pesquisa era explicada e, cada participante assinava o Termo de Consentimento Livre e Esclarecido (TCLE), caso desejasse participar. Posteriormente, a entrevista inicial era respondida e o pesquisador apresentava a FAB, explicando as instruções de cada subteste por vez.

É importante salientar que como se trata de uma bateria com muitos itens, fez-se necessário atentar para possíveis variáveis intervenientes. Para evitar a influência de sons, músicas e conversas que possam influenciar na interpretação afetiva e, consequentemente, na escolha correta para cada item, a estabilidade do setting de aplicação foi garantida, sendo utilizado sempre um ambiente iluminado e silencioso. Alguns participantes reclamaram da aparente semelhança entre os itens, o que poderia gerar monotonia e diminuir o engajamento na atividade. Para contornar tais efeitos, realizou-se uma pausa de 5 minutos entre os subtestes, onde o participante poderia transmitir sua impressão sobre as tarefas, bem como desfocar a atenção dos estímulos.

A bateria foi aplicada com o auxílio de um computador portátil Acer Aspire 4740, em uma apresentação de slides, contendo os estímulos do instrumento, sendo a parte auditiva assessorada por um par de mini caixas de som para notebooks, com potência de quatro watts RMS. As respostas foram marcadas em folha-resposta padronizada, semelhante à original.

\section{Análise estatística dos dados}

Os resultados foram analisados estatisticamente, através de métodos paramétricos, em busca da congruência na resposta dos participantes, verificando-se a porcentagem de acertos em cada subteste e em todo o teste. A média da população nacional, obtida em cada subteste, foi comparada, através de testes $t$, com a média da amostra estadunidense, para a mesma faixa etária. Foram comparadas as médias de acerto da população brasileira entre homens e mulheres para cada subteste, também através de testes $t$, em busca de possíveis efeitos de gênero. Para checar os efeitos da escolaridade, a ANOVA foi utilizada para comparação entre as médias dos subgrupos superior incompleto, superior completo e pós-graduação. Ressalta-se que a escolha de testes paramétricos deu-se, apesar do efeito-teto nas respostas dos subtestes (que poderia ocasionar violação dos parâmetros de normalidade), pois sabe-se que, ainda assim, são robustos e produzem resultados fidedignos (Boneau, 1960; Posten, 1978; Rasch \& Guiard, 2004). Dado o caráter preliminar do estudo, uma análise de componentes principais também foi realizada, a fim de identificar os fatores latentes associados aos subtestes.

\section{Resultados}

O desempenho da amostra brasileira nos subtestes aponta índices muito altos de acertos para os diferentes subtestes, variando entre $84,8 \%$, no subteste 2 e $97,6 \%$, no subteste 1 (ambos subtestes avaliam reconhecimento de identidade/ semelhança de faces e de expressões faciais), indicando bom reconhecimento das diferentes expressões, bem como da prosódia emocional associada, conforme apresentado na figura 1.

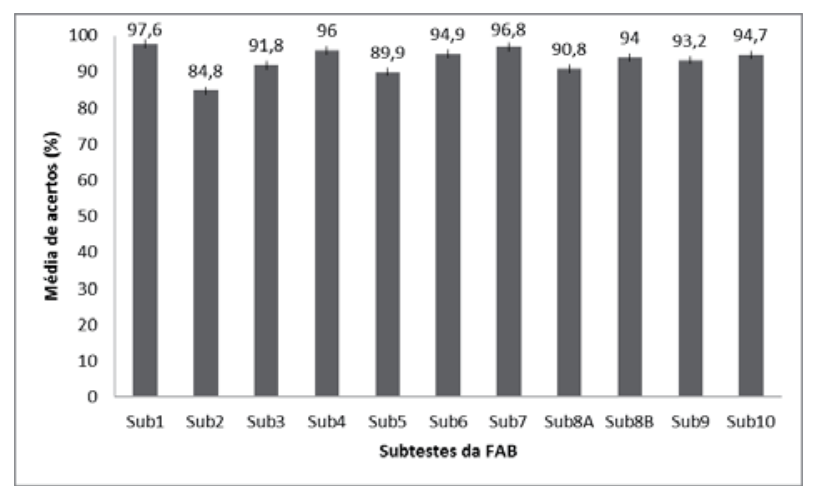

Figura 1. Média de Acertos da Amostra Populacional Brasileira (em Porcentagem) para os Subtestes da FAB

Nesta mesma figura, percebe-se a homogeneidade da resposta por parte da amostra, que mesmo em subtestes que exigem maior carga cognitiva como os subtestes $8 \mathrm{~B}$ (semântica e prosódia conflitante), 9 (combinação prosódia-expressão facial) e 10 (combinação expressão facial-prosódia), atingiram altos índices de acerto, respectivamente: 94\%, 93,2\% e 94,7\%.

$\mathrm{Na}$ comparação do desempenho avaliativo entre homens e mulheres não houve diferença significativa entre as médias dos subtestes, para $t_{\text {crit }}(67)=1,996$, com $p<0,05$, ambos os grupos com altas taxas de acerto, não configurando um efeito da variável gênero no processo de reconhecimento de emoções (Tabela 1). 
Tabela 1

Comparação das Médias dos Subgrupos Masculino e Feminino para a Amostra Brasileira

\begin{tabular}{|c|c|c|c|c|c|c|}
\hline \multirow[b]{3}{*}{$\begin{array}{l}\text { Tarefas } \\
\text { da FAB }\end{array}$} & \multicolumn{4}{|c|}{ Gênero } & \multirow[b]{3}{*}{$\begin{array}{c}\text { tcrit(67) }= \\
1,996\end{array}$} & \multirow[b]{3}{*}{$\begin{array}{l}p< \\
0,05\end{array}$} \\
\hline & \multicolumn{2}{|c|}{ Feminino } & \multicolumn{2}{|c|}{ Masculino } & & \\
\hline & Média & $D P$ & Média & $D P$ & & \\
\hline Sub1 & 97,5 & $( \pm 4,5)$ & 97,9 & $( \pm 4,7)$ & $t=-0,383$ & ns \\
\hline Sub2 & 85,5 & $( \pm 6,7)$ & 83,9 & $( \pm 9,3)$ & $t=0,789$ & ns \\
\hline Sub3 & 92,6 & $( \pm 5,6)$ & 90,8 & $( \pm 8,7)$ & $t=1,014$ & ns \\
\hline Sub4 & 96 & $( \pm 6,3)$ & 96 & $( \pm 7,7)$ & $t=-0,020$ & ns \\
\hline Sub5 & 90,5 & $( \pm 10,3)$ & 89,1 & $( \pm 10,7)$ & $t=0,530$ & ns \\
\hline Sub6 & 94,2 & $( \pm 5,1)$ & 95,8 & $( \pm 3,7)$ & $t=-1,390$ & ns \\
\hline Sub7 & 96,2 & $( \pm 6,3)$ & 97,7 & $( \pm 3,9)$ & $t=-1,127$ & ns \\
\hline Sub8A & 90,5 & $( \pm 10,6)$ & 91,3 & $( \pm 8)$ & $t=-0,374$ & ns \\
\hline Sub8B & 93,5 & $( \pm 9)$ & 94,5 & $( \pm 6,5)$ & $t=-0,509$ & ns \\
\hline Sub9 & 93,1 & $( \pm 6,7)$ & 93,4 & $( \pm 6,9)$ & $t=-0,194$ & ns \\
\hline Sub10 & 94,2 & $( \pm 7,2)$ & 95,3 & $( \pm 5,5)$ & $t=-0,690$ & ns \\
\hline
\end{tabular}

Nota. ns-não significativo para $\mathrm{p}<0,05$

Resultado semelhante foi encontrado na comparação da média de desempenho entre as diferentes escolaridades. A única diferença significativa encontrada entre estes grupos foi no subteste 6 , de diferenciação de prosódia não-emocional, onde a média de acertos dos participantes com nível superior incompleto foi significativamente inferior à média dos participantes com nível superior completo ou pós-graduados, para $F(2,66)=26,750$, com $p<0,05$ (Tabela 2).

Tabela 2

Comparação das Médias dos Subgrupos por Escolaridade para a Amostra Brasileira

\begin{tabular}{|c|c|c|c|c|c|c|c|}
\hline & & & Esco & ridade & & & \\
\hline & Superior & completo & Superio & Completo & Pós-g & duação & \\
\hline $\begin{array}{l}\text { Tarefas } \\
\text { da FAB }\end{array}$ & Média & $D P$ & Média & $D P$ & Média & $D P$ & $\begin{array}{l}P< \\
0,05\end{array}$ \\
\hline Sub1 & 97,1 & $( \pm 5)$ & 98,07 & $( \pm 3,8)$ & 100 & $( \pm 0,0)$ & ns \\
\hline Sub2 & 84,6 & $( \pm 7,3)$ & 86,5 & $( \pm 11,4)$ & 83,3 & $( \pm 5)$ & ns \\
\hline Sub3 & 90,9 & $( \pm 7,7)$ & 95 & $( \pm 4,5)$ & 92 & $( \pm 5,6)$ & ns \\
\hline Sub4 & 95 & $( \pm 7,7)$ & 98,4 & $( \pm 3,1)$ & 97,7 & $( \pm 5)$ & ns \\
\hline Sub5 & 87,34 & $( \pm 11)$ & 95,3 & $( \pm 7,2)$ & 95,5 & $( \pm 5,2)$ & ns \\
\hline Sub6 & 92,7 & $( \pm 4)$ & 99,51 & $( \pm 1,7)$ & 94,9 & $( \pm 4,6)$ & s \\
\hline Sub7 & 96,3 & $( \pm 5,8)$ & 98 & $( \pm 2,5)$ & 97,7 & $( \pm 6,6)$ & ns \\
\hline Sub8A & 88,4 & $( \pm 10,4)$ & 95,3 & $( \pm 4,3)$ & 97,2 & $( \pm 3,6)$ & ns \\
\hline Sub8B & 91,6 & $( \pm 8,78)$ & 98,9 & $( \pm 1,4)$ & 99 & $( \pm 2)$ & ns \\
\hline Sub9 & 91,8 & $( \pm 7,1)$ & 95,3 & $( \pm 5,5)$ & 97,7 & $( \pm 3,6)$ & ns \\
\hline Sub10 & 93,7 & $( \pm 7,3)$ & 96,5 & $( \pm 4,2)$ & 97,2 & $( \pm 3,6)$ & ns \\
\hline
\end{tabular}

Nota. ns-não significativo para $p<0,05$. s- significativo para $p<0,05$, com sig $<0,01$
Na comparação entre os resultados das amostras das duas populações (Brasil e EUA), embora os resultados dos estadunidenses tenham sido ligeiramente superiores, as diferenças entre as médias não foram consideradas significativas para $\mathrm{t}_{\text {crit }}(110)=1,657$, com $p<0,05$, conforme apresentado na Tabela 3 .

Tabela 3

Distribuição Comparativa das Médias de Desempenho da FAB por Subteste para Amostra Brasileira e Estadunidense

\begin{tabular}{|c|c|c|c|c|c|c|}
\hline & \multicolumn{4}{|c|}{ Nacionalidade } & \multirow[b]{3}{*}{$\operatorname{tcrit}(110)=1,667$} & \multirow[b]{3}{*}{$p<0,05$} \\
\hline & \multicolumn{2}{|c|}{ Brasileiros } & \multicolumn{2}{|c|}{ Estadunidense } & & \\
\hline $\begin{array}{l}\text { Tarefas } \\
\text { da FAB }\end{array}$ & Média & $D P$ & Média & $D P$ & & \\
\hline Sub1 & 97,6 & $( \pm 4,5)$ & 97,7 & $( \pm 3,9)$ & $t=-0,02$ & ns \\
\hline Sub2 & 84,8 & $( \pm 7,9)$ & $92,4)$ & $( \pm 7,3)$ & $t=-0,69$ & ns \\
\hline Sub3 & 91,8 & $( \pm 7,1)$ & 94,7 & $( \pm 5,9)$ & $t=-0,348$ & ns \\
\hline Sub4 & 96 & $( \pm 6,8)$ & 98,5 & $( \pm 2,7)$ & $t=-0,378$ & ns \\
\hline Sub5 & 89,2 & $( \pm 10,4)$ & 96,7 & $( \pm 5,7)$ & $t=-0,436$ & ns \\
\hline Sub6 & 94,9 & $( \pm 4,6)$ & 99,5 & $( \pm 1,6)$ & $t=-1,53$ & ns \\
\hline Sub7 & 96,8 & $( \pm 5,4)$ & 99,6 & $( \pm 1,8)$ & $t=-0,652$ & ns \\
\hline Sub8A & 90,8 & $( \pm 9,5)$ & 96,7 & $( \pm 5,1)$ & $t=-0,422$ & ns \\
\hline Sub8B & 94 & $( \pm 8)$ & 89,5 & $( \pm 8,6)$ & $t=-0,392$ & ns \\
\hline Sub9 & 93,2 & $( \pm 6,7)$ & 98,2 & $( \pm 4,1)$ & $t=-0,741$ & ns \\
\hline Sub10 & 94,7 & $( \pm 6,5)$ & 99,4 & $( \pm 1,5)$ & $t=-0,787$ & ns \\
\hline
\end{tabular}

Nota. ns-não significativo para $p<0,05$

Ambas as amostras apresentaram altos índices de acerto, confirmando um efeito-teto, já que o objetivo dos subtestes da FAB é identificar dificuldades associadas ao processo de reconhecimento emocional (Bowers, Blonder, \& Heilman, 1999).

Como a Florida Affect Battery, em seu manual, afirma ser bifatorial (visual e auditiva), conduziu-se uma análise de componentes principais com dois fatores fixos. A adequação da amostra foi razoável $(\mathrm{KMO}=0,73)$. O teste de esfericidade de Bartlett $\left[\chi^{2}(55)=271,52, p<0,001\right]$, indicou que as correlações entre os itens eram suficientes para a realização da análise. Esta análise demonstrou que os dois componentes obedeceram ao critério de Kaiser (autovalor maior que 1), explicando apenas $49,1 \%$ da variância. Segundo a matriz de componente rotativa obtida, o componente 1 seria formado pelos subtestes 7, 8A, 8B, 9 e 10 e o componente 2, pelos subteste 1,2 ,4 e 5 (Figura 2). 


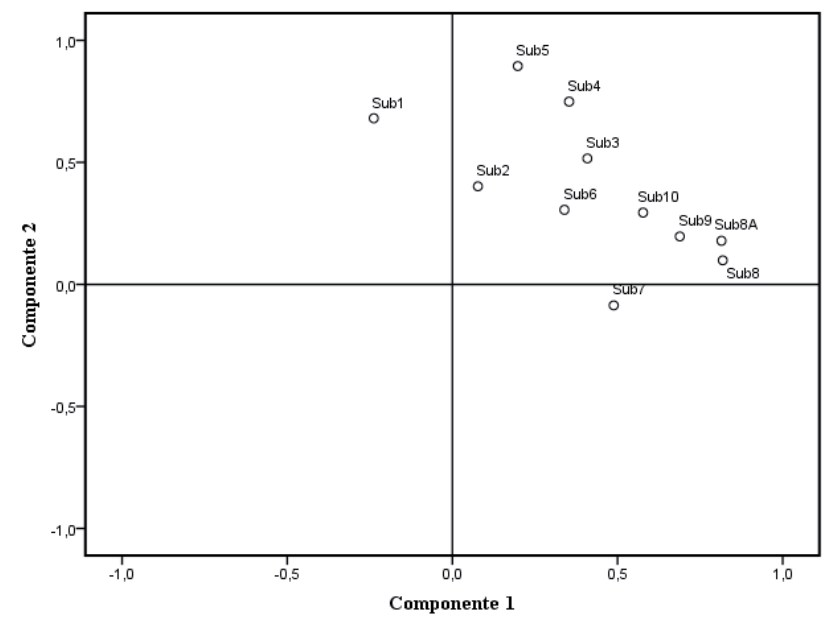

Figura 2. Gráfico dos Subtestes da FAB Associados aos Componentes 1 e 2, para Análise de Componentes Principais, com Dois Fatores.

O subteste 6 não estaria associado a nenhum componente e o subteste 3 estaria associado aos dois componentes.

No entanto, ao se avaliar os autovalores iniciais da variância explicada $(3,83 ; 1,54 ; 1,22$ e 1,18$)$ e o gráfico de sedimentação (Figura 3), quatro fatores emergiram, explicando, 70,9\% da variância, sugerindo que a bateria, possivelmente, não poderia ser classificada como bifatorial.

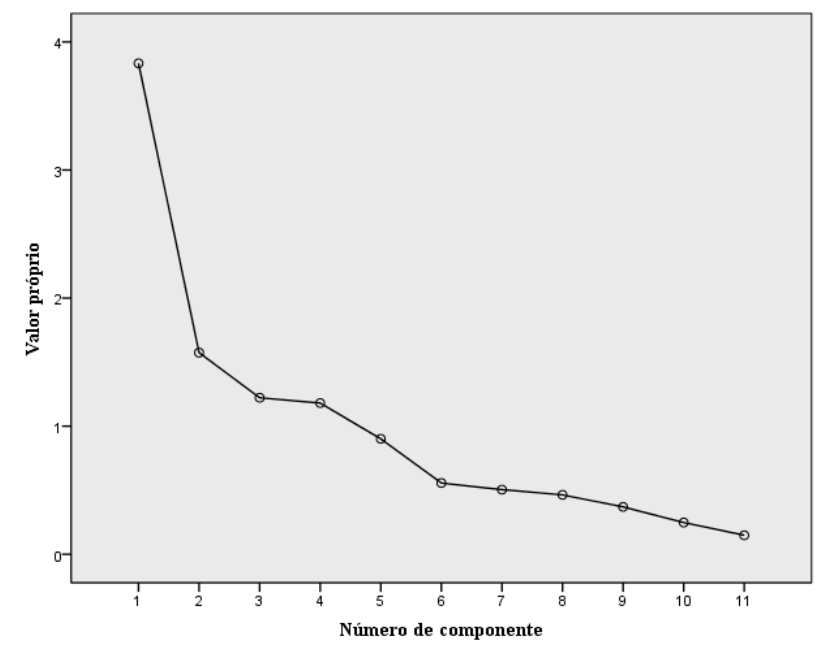

Figura 3. Gráfico de Sedimentação para Análise de Componentes Principais da FAB

Ao realizar uma segunda análise de fatores, respeitando o critério de Kaiser, e sem a pré-determinação do número de componentes, efetivamente foram encontrados quatro componentes, a saber: subtestes $6,8 \mathrm{~A}, 8 \mathrm{~B}$ e 9 integrariam o componete 1 ; subtestes 1 , 4 e 5 integrariam o componente 2 ; subtestes 2 e 3 integrariam o componente 3 , e subtestes 7 e 10 integraria o componente 4.

\section{Discussão}

Conforme Ekman e Friesen (1987), raiva, tristeza, alegria e medo, são emoções reconhecidas e expressadas universalmente, juntamente com surpresa e nojo. Sabe-se que o poder de reconhecimento e expressão pode ser mediado pela exposição às regras culturais que estimulam ou dificultam essas habilidades (Elfenbein, 2013). No entanto, a presente pesquisa corrobora pesquisas anteriores (Beaupre, 2005; Biehl et al., 1997; Ekman, 1972; Ekman \& Friesen, 1969; Matsumoto, 1990, 1992; Matsumoto \& Hwang, 2011; Matsumoto, Hwang, \& Yamada, 2010; Sauter et al, 2010), que apontam para altos índices de reconhecimento de expressões faciais semelhantes em diferentes culturas.

Para a prosódia emocional, o resultado da bateria se torna ainda mais expressivo, dada a escassez de instrumentos nacionais para avaliá-la. Por ser um aspecto bastante influenciado pelo contexto cultural, seria de grande interesse expandir esta avaliação para outras regiões do país, verificando a existência ou não de diferenças significativas, haja vista a variabilidade de sotaques, entonações e expressões vocais de emoção.

A tradução e adaptação de uma bateria que possibilita avaliar os dois principais aspectos não-verbais da afetividade de maneira integrada é de grande importância não só para o contexto da pesquisa, como também para a prática clínica. Este estudo exploratório demonstrou a sua aplicabilidade em uma amostra populacional brasileira, cabendo normatizações e validações posteriores.

Por ser uma bateria de avaliação de dificuldades de reconhecimento emocional, devido à sua especificidade e variedade de tarefas, a FAB aparenta ser um instrumento interessante para uso neuropsicológico, auxiliando na identificação de patologias do reconhecimento afetivo e também perceptual.

A questão da diferença entre os sexos no reconhecimento de emoção ainda não possui um consenso, com estudos apontando para diferenças no desempenho quando consideramos o gênero (Besson, Magne, \& Schön, 2002; Biele \& Grabowska, 2006; Elfenbein \& Ambady, 2002; Hall \& Matsumoto, 2004; Kret \& De Gelder, 2012; Thayer \& Johnsen, 2000), embora Vassallo, Cooper e Douglas (2009) tenham encontrado médias de reconhecimento semelhantes em faces emocionais, em um estudo com eyetrackers, sendo as diferenças visíveis, possíveis estratégias de reconhecimento diferenciadas, bem como tempo de latência e áreas de interesse acessadas. Assim, o presente contribui com mais um resultado em relação aos gêneros, evidenciando a ausência de diferenças significantes entre o desempenho de homens e mulheres jovens, onde ambos apresentaram excelentes índices de acerto, tanto para prosódia como para expressões faciais.

A ausência de diferenças significativas entre as médias por escolaridade também já era esperada, dado o caráter evolutivo das emoções básicas. Estudos apontam para o bom reconhecimento de emoções em crianças pequenas, mesmo entre aquelas não inseridas ainda no contexto escolar (Elfenbein \& Ambady, 2002; Kestenbaum \& Nelson, 1992; MacDonald, Kirpatrick, \& 
Sullivan, 1996; McClure, 2000). No entanto, seria interessante a expansão das faixas de escolaridade, para comparação, e para constatação da adequação da Florida Affect Battery em diferentes amostras da população.

Apesar da bifatorialidade (tarefas visuais/auditivas) afirmada no manual da FAB, a análise fatorial conduzida, aparentemente, sugere a existência de quatro componentes. Poderiase pensar nos objetivos de avaliação das tarefas como sendo estes quatro fatores: reconhecimento de identidade facial, reconhecimento de expressão facial, reconhecimento de prosódia não-emocional e reconhecimento de prosódias emocionais. No entanto, considerando o modo como os subtestes se dividem e seus objetivos, não é o que ocorre. Ao associar os componentes encontrados e os objetivos das tarefas, não se encontram correspondências efetivamente preditivas, que pudessem sustentar um constructo. Vale ressaltar que tais dados são exploratórios e preliminares e, por isso, novas pesquisas precisam ser conduzidas, incluindo um número maior de participantes por variável, de modo a minimizar qualquer efeito possível do tamanho da amostra. O intuito desta pesquisa não foi normatizar ou validar a FAB, mas sim, adaptá-la à população brasileira e explorar preliminarmente os dados obtidos, auxiliando a fornecer diretrizes para normatizações futuras.

\section{Considerações finais}

Do conhecimento das autoras, este é o primeiro relato de pesquisa utilizando a Florida Affect Battery no Brasil, sendo seus estímulos considerados preliminarmente apropriados para o uso na população brasileira. Estudos futuros são necessários para a normatização da bateria. No entanto, este estudo preliminar já aponta para a adequação dos estímulos visuais, bem como dos conteúdos semânticos e da estruturação da bateria. No geral, a amostra da população brasileira avaliada apresentou o resultado esperado, com altos índices de reconhecimento de expressões faciais e prosódia emocional, sem diferença significativa entre o desempenho de homens e mulheres, entre escolaridades e sem diferenças significativas com a amostra populacional estadunidense. Tal resultado, mais uma vez, fornece indícios para a teoria das emoções básicas, com altos índices de reconhecimento de expressões faciais universais em diferentes culturas. Futuramente, seria interessante ampliar a amostra do estudo, considerando outras variáveis como diferentes idades, escolaridades, localidade (diferentes estados ou localidades habitacionais, haja vista uma possível interferência na prosódia), bem como em condições clínicas que apresentam prejuízos no reconhecimento de emoções através de sinais não-verbais, verificando além do desempenho, a viabilidade de aplicação da Florida Affect Battery nestes grupos. Com uma amostra maior, outros tipos de análise de fatores, como a confirmatória, posteriormente a uma nova análise exploratória, poderiam ser realizadas, identificando os construtos realmente acessados pela bateria e fornecendo maior segurança e validade aos resultados obtidos.

\section{Referências}

Beaton, D., Bombardier, C., Guillemin, F., \& Ferraz, M. (2000). Guidelines for the process of cross-cultural adaptation of self-report measures. Spine, 25(24), 3186-3191.

Beaupre, M. (2005). Cross-cultural emotion recognition among canadian ethnic groups. Journal of Cross-Cultural Psychology, 36(3), 355-370. doi: $10.1177 / 0022022104273656$

Besson, M., Magne,C., \& Schön, D. (2002). Emotional prosody: Sex differences in sensitivity to speech melody. Trends in Cognitive Sciences, 6(10), 405407. doi: 10.1016/S1364-6613(02)01975-7

Biehl, M., Matsumoto, D., Ekman, P., Meant, V., Heider, K., Kudoh, T., \& Ton, V. (1997). Matsumoto and Ekman's japanese and caucasian facial expressions of emotion: Reliability data and cross-national differences. Journal of Nonverbal Behavior, 21(1), 3-21. doi: 10.1023/A:1024902500935

Biele, C., \& Grabowska, A. (2006). Sex differences in perception of emotion intensity in dynamic and static facial expressions. Experimental Brain Research, 171(1), 1-6. doi: 10.1007/s00221-005-0254-0

Boneau, C. A. (1960). The effects of violation of assumptions underlying the t-test. Psychological Bulletin, 57(1), 49-64. doi: 10.1037/h0041412

Bowers, D., Bauer, R., \& Heilman, K. (1993). The nonverbal affect lexicon: Theoretical perspectives from neuropsychological studies of affect perception. Neuropsychology, 7(4), 733-744. doi: 10.1037/08944105.7.4.433

Bowers, D., Blonder, L., \& Heilman K. (1999). The Florida affect battery. Center of Neuropsychological Studies, University of Florida, Gainesville, FL.

Bucks, R., \& Radford,A. (2004). Emotion processingin Alzheimer's disease.Aging \& Mental Health, 8(3), 222-232. doi: 10.1080/13607860410001669750

Campanella, S., \& Belin, P. (2007). Integrating face and voice in person perception. Trends in Cognitive Science, 11(12), 535-543. doi:10.1016/j. tics.2007.10.001

Dael, N., Mortillaro, M., \& Scherer, K. R. (2012). Emotion expression in body action and posture. Emotion, 12(5), 1085-101. doi: 10.1037/a0025737

Darwin, C. (1998). The expression of the emotions in man and animals (3a ed.). Nova Iorque: Oxford University Press.

Ekman, P. (1972). Universals and cultural differences in facial expression of emotion. In J. Cole (Org.), Nebraska Symposium on Motivation (vol. 19). Nebraska: Lincoln University of Nebraska Press.

Ekman, P. (1992). Are there basic emotions? Psychology Review, 99(3), 550553. doi: $10.1037 / 0033-295 X .99 .3 .550$

Ekman, P. (1999). Basic emotions. In T. Dalgleiish \& M. Power (Orgs.), Handbook of Cognition and Emotion. Sussex: Wiley \& Sons.

Ekman, P. (2003). Emotions Revealed. Nova Iorque, USA: Henry Holt and Company.

Ekman, P., \& Cordaro, D. (2011). What is meant by calling emotions basic. Emotion Review, 3(4), 364-370. doi: 10.1177/1754073911410740

Ekman, P., \& Friesen, W. (1969). The repertoire of non verbal behavior: Categories, origins, usage, and coding. Semiotica, 1(1), 49-98. doi: 10.1515/9783110880021

Ekman, P., \& Friesen, W. (1987). Universals and cultural differences in the judgments of facial expression of emotion. Journal of Personality and Social Psychology, 53(4), 712-717. doi: 10.1037/0022-3514.53.4.712

Elfenbein, H. (2013). Nonverbal dialects and accents in facial expressions of emotion. Emotion Review, 5(1), 90-96. doi:10.1177/1754073912451332

Elfenbein, H., \& Ambady, N. (2002). On the universality and cultural specificity of emotion recognition: A meta-analysis. Psychological Bulletin, 128(2), 203-235. doi: 10.1037//0033-2909.128.2.203

Hall, J., \& Matsumoto, D. (2004). Gender differences in judgments of multiple emotions from facial expressions. Emotion, 4(2), 201-206. doi: 10.1037/1528-3542.4.2.201 
Hambleton, R., Merenda, P., \& Spielberger, C. (2005). Adapting educational and psychological tests for cross-cultural assessment. Nova Jersey: LEA.

International Test Commission (2000). International Guidelines for Translating and Adapting Tests. Recuperado de www.intestcom.org/itc_projects.htm

Izard, C. (2010). The many meanings/aspects of emotion: Definitions, functions, activation, and regulation. Emotion Review. 2(4), 363-370. doi: $10.1177 / 1754073910374661$

Kestenbaum, R., \& Nelson, C. (1992). Neural and behavioural correlates of emotion recognition in children and adults. Journal of Experimental Child Psychology, 54(1), 1-18. doi: 10.1016/0022-0965(92)90014-W

Kret, M., \& De Gelder, B. (2012). A review on sex differences in processing emotional signals. Neuropsychologia, 50(7), 1211-1221. doi:10.1016/j. neuropsychologia.2011.12.022

MacDonald, P., Kirkpatrick, S., \& Sullivan, L. (1996). Schematic drawings of facial expressions for emotion recognition and interpretation by preschool-aged children. Genetic, Social, and General Psychology Monographs, 122(4), 373-388.

Ma-Kellams, C., \& Blascovich, J. (2012). Inferring the emotions of friends versus strangers: The role of culture and self-construal. Personality \& Social Psychology Bulletin, 38(7), 933-945. doi:10.1177/0146167212440291

Marian, D., \& Shimamura, A. (2012). Emotions in context: Pictorial influences on affective attributions. Emotion, 12(2), 371-375. doi: 10.1037/a0025517

Matsumoto, D. (1990). Cultural similarities and differences in display rules. Motivation and Emotion, 14(3), 195-214. doi:10.1007/BF00995569

Matsumoto, D. (1992). American-Japanese cultural differences in the recognition of facial expressions. Journal of Cros-Cultural Psychology, 23(1), 72-84. doi: 10.1177/0022022192231005

Matsumoto, D., \& Hwang, H. S. (2011). Evidence for training the ability to read microexpressions of emotion. Motivation and Emotion, 35(2), 181191. doi:10.1007/s11031-011-9212-2

Matsumoto, D., Hwang, H. S., \& Yamada, H. (2010). Cultural differences in the relative contributions of face and context to judgments of emotions. Journal of Cross-Cultural Psychology, 43(2), 198-218. doi:10.1177/0022022110387426

McClure, E. (2000). A meta-analytic review of sex differences in facial expression processing and their development in infants, children, and adolescents. Psychological Bulletin, 126(3), 424-453. doi: 10.1037/00332909.126.3.424

Organização Mundial de Saúde. (2010). Process of translation and adaptation of instruments. Recuperado de //www.who.int/substance_abuse/research_ tools/ translation/en/

Paulman, S., Titone, D., \& Pell, M. (2012). How emotional prosody guides your way: Evidence from eye movements. Speech Comunication, 54(1), 92-107. doi:10.1016/j.specom.2011.07.004

Posten, H. O. (1978). The robustness of the two sample t-test over the Pearson system. Journal of Statistical Computation and Simulation, 6(3-4), 295-311. doi: 10.1080/00949657808810197

Rasch, D., \& Guiard, V. (2004). The robustness of parametric statistical methods. Psychology Science, 46(7), 175-208. doi: 10.1037/0003066X.63.7.591

Regenbogen, C., Schneider, D., Gur, R., Schneider, F., Habel, U., \& Kellermann, T. (2012). Multimodal human communication - targeting facial expressions, speech content and prosody. NeuroImage, 60(4), 2346-56. doi:10.1016/j.neuroimage.2012.02.043

Rigoulot, S., \& Pell, M. D. (2012). Seeing emotion with your ears: Emotional prosody implicitly guides visual attention to faces. PLOS One, 7(1), e30740. doi:10.1371/journal.pone.0030740

Sauter, D., Eisner, F., Ekman, P., \& Scott, S. K. (2010). Cross-cultural recognition of basic emotions through nonverbal emotional vocalizations. Proceedings of the National Academy of Sciences of the United States of America, 107(6), 2408-12. doi:10.1073/pnas.0908239106
Scherer, K., Banse, R., \& WallBott, H. (2001). Emotion inferences from vocal expression correlate across languages and cultures. Journal of CrossCultural Psychology, 32(1), 76-92. doi: 10.1177/0022022101032001009

Testa, J., Beatty, W., Gleason, A., Orbelo, B., \& Ross, E. (2001). Impaired affective prosody in AD: Relationship to aphasic deficits and emotional behaviors. Neurology, 57(8), 1474-1481. doi: 10.1212/WNL.57.8.1474

Thayer, J., \& Johnsen, B. (2000). Sex differences in judgement of facial affect: A multivariate analysis of recognition errors. Scandinavian Journal of Psychology, 41(3), 243-246. doi: 10.1111/1467-9450.00193

Tracy, J. L., \& Randles, D. (2011). Four models of basic emotions: A review of Ekman and Cordaro, Izard, Levenson, and Panksepp and Watt. Emotion Review, 3(4), 397-405. doi:10.1177/1754073911410747

Vassallo, S., Cooper, S. L., \& Douglas, J. M. (2009). Visual scanning in the recognition of facial affect : Is there an observer sex difference? Journal of Vision, 9(3), 1-10. doi:10.1167/9.3.11.

Whalen, P. J., Raila, H., Bennett, R., Mattek, A., Brown, A., Taylor, J., \& Palmer, A. (2013). Neuroscience and facial expressions of emotion: The role of amygdala-prefrontal interactions. Emotion Review, 5(1), 78-83. doi:10.1177/1754073912457231 
Hélida Arrais Costa-Vieira, Mestre em Ciências do Comportamento (Cognição e Neurociências) pela Universidade de Brasília (UnB), é Doutoranda em Ciências do Comportamento (Cognição e Neurociências) na Universidade de Brasília (UnB). Endereço para correspondência: Departamento de Processos Psicológicos Básicos, Instituto de Psicologia, Universidade de Brasília; Caixa Postal 4500; 70910-900 Brasília, DF. Telefone: (61) 3107-6833. E-mail: helida.costavieira@gmail.com

Wânia Cristina de Souza, Doutora em Medicina na Toyama Medical and Pharmaceutical University TMPU Toyama-Japan, Pós-Doutora em Percepção Visual na Universidade de Brasília, é vice-diretora do Instituto de Psicologia da Universidade de Brasília (UnB). E-mail: wcdeep@gmail.com 